\title{
El debate sobre el cambio climático interpretado como un juego de persuasión *
}

(The climate change debate as a persuasion game)

Leonardo MONZONÍs FORNER y Jesús ZAMORA BONILLA

Received: 24.10.2011

Final version: 21.2.2012

BIBLID [0495-4548 (2013) 28: 76; pp. 77-96]

RESUMEN: Los científicos tratan de persuadir a sus colegas y, en última instancia, al conjunto de la sociedad, para que acepten sus tesis, descubrimientos y propuestas. Desarrollan para ello una serie de estrategias que pueden ser estudiadas como un "juego de persuasión” en el que intervienen, además de los procesos de argumentación formal e informal típicamente estudiados por la lógica y metodología de la ciencia, aspectos tradicionalmente considerados "sociológicos". En este artículo se analiza el debate sobre la ciencia del cambio climático como un juego de este tipo, desde un enfoque inferencialista, sobre la base del esquema presentado en Zamora Bonilla (2006a).

Palabras clave: cambio climático; persuasión; racionalidad; inferencialismo; debates científicos; cambio científico; argumentación: evaluación de argumentos.

ABSTRACT: Scientists try to persuade their colleagues, and ultimately the whole society, of the acceptability of their claims, discoveries and proposals. In order to reach that goal, they develop a number of strategies that can be studied as a 'game of persuasion', in which, besides the processes of formal and informal argumentation typically studied by logic and methodology of science, there are also 'sociological' aspects intervening. This paper analyses the debate on climate change science as a 'persuasion game', from an inferentialist point of view, according to the lines of Zamora Bonilla (2006a).

Keywords: climate change; persuasion; rationality; inferentialism; scientific debates; scientific change; argumentation; evaluation of arguments.

\section{El caso de los correos filtrados y el problema de la aceptación o rechazo de la Teoría del Calenta- miento Global}

En noviembre de 2009, saltó a los medios de comunicación la noticia de que alguien desconocido había accedido al servidor de la Unidad de Investigación Climática (URC) de la Universidad de East Anglia, en Norwich, Inglaterra, y que se habían filtrado 160 MB de datos, conteniendo correos electrónicos y otros tres mil documentos. De entre todo el material filtrado, que ha sido reconocido como auténtico, algunos correos han resultado especialmente polémicos. Uno de ellos, de noviembre de 1999, se refería a un gráfico ${ }^{1}$ que representaba la evolución de la temperatura media del hemisferio norte del último milenio, que tenía que figurar en un informe de la OMM. En el correo se

\footnotetext{
* Agradecemos el apoyo del proyecto de investigación FFI2011-23267.

${ }^{1}$ El gráfico representa las temperaturas medias del Hemisferio Norte entre el año 1000 y el 2000. Contiene datos de registros instrumentales desde 1850, pero los datos anteriores a 1850 son datos proxy, reconstruidos a partir de la variación en anchura de los anillos de algunos árboles, por ejemplo. Esta reconstrucción y el acoplamiento de unos datos y otros es el principal motivo de las controversias.
} 
decía: "He completado el truco de Mike en Nature ${ }^{2}$ [...] para esconder el descenso"3. Otros correos polémicos fueron enviados entre los años 2003 y 2009, y se referían a asuntos tales como la legitimidad y competencia de algunos científicos para participar en la revisión entre colegas; y en otros, parecía deducirse la voluntad de esquivar los requerimientos de la Ley de Libertad de Información británica. A partir del conocimiento de la filtración se abrió un debate en torno a varios aspectos del asunto: sobre el mismo acto de piratería informática, sobre la honestidad de los científicos de la URC, sobre el descrédito o no de la Teoría del Calentamiento Global (TCG), sobre la influencia de estos hechos en la cumbre de Copenhague, etc.

G. Monbiot ${ }^{4}$, por ejemplo, abordó la cuestión del posible descrédito de la TCG unos días después de que se conociera la filtración. Para ello utilizó el recurso literario de redactar el correo que hubiera necesitado leer para poder afirmar que la Teoría del Calentamiento Global es una teoría desacreditada. Este correo, decía Monbiot, tendría que haber mostrado una conspiración prolongada durante 185 años, conspiración en la que el primer participante habría sido Fourier, que habría continuado con Tyndall, Svante Arrhenius y Max Plank; científicos que han aportado las teorías físicas básicas sobre las que se sustenta la Teoría del Calentamiento Global. El correo tendría que haber puesto también de manifiesto una conspiración para controlar el registro instrumental de temperaturas y puesto en evidencia que el debilitamiento de la capa de hielos árticos es en realidad el resultado de maniobras secretas no detectadas, así como también lo es la migración de las aves. Debería mostrar también que los gobiernos han abrazado la causa del calentamiento global bajo amenazas y chantajes y que las academias nacionales de ciencias lo han hecho como consecuencia de sobornos. Efectivamente, este tipo de conspiración no es el que aparece en los correos filtrados, y por ello concluye Monbiot que estos solamente dañan la credibilidad personal de tres o cuatro científicos, y afectan a una o dos cuestiones puntuales de las cientos de evidencias que avalan la Teoría del Calentamiento Global.

Esta es ciertamente una respuesta brillante, pero ¿es suficiente con las evidencias empíricas y la cobertura de la teoría del efecto invernadero para zanjar el debate sobre la teoría del calentamiento global, ¿qué hace falta para que una teoría científica sea aceptada o rechazada?

Durante gran parte de siglo XX, los filósofos de la ciencia han dedicado no pocos esfuerzos a tratar de responder a esta cuestión. Así, el falsacionismo de Popper, la versión refinada de Lakatos, o las nociones de paradigma y de revolución científica de Kuhn, han tratado de dar cuenta del modo en que la ciencia progresa. Uno de los hilos

\footnotetext{
${ }^{2}$ Se refiere al artículo de Nature de 23 de abril de 1998, de Mann, Bradley y Hughes: "Global-scale temperature patterns and climate forcing over the past six centuries".

${ }^{3} \mathrm{El}$ texto completo, traducido al castellano, es el siguiente: "Acabo de completar el truco de Mike en $\mathrm{Na}$ ture agregando las temperaturas reales a cada una de las series de los últimos 20 años (desde 1981 en adelante) y desde 1961 para que Keith esconda la declinación. Las series de Mike tiene los valores marinos y de tierra mientras que los otros dos tienen Abril-Sept para la tierra del HN al N de 20N. Las últimas dos son reales para 1999, mientras que la estimación para 1999 para el HN combinado es +44 wrt 61-90. El estimado para 1999 con datos hasta oct es *0,35 cf. 0,57 par 1998."

4 “Global warming rigged? Here's the mail I'd need to see" (Monbiot 2009).
}

Theoria 76 (2013): 77-96 
conductores de estas reflexiones es precisamente lo problemático del arbitraje de la experiencia. Para Lakatos, el obstáculo principal para que un experimento sea determinante es que valorar un experimento que pretenda refutar una teoría requiere una especie de racionalidad instantánea capaz de evaluar todas y cada una de las implicaciones que se deriven de ese experimento. De hecho, un resultado experimental negativo puede ser bloqueado por la tenacidad de los defensores de una teoría, que tienen a su disposición distintos modos de desacreditarlo: mediante la crítica de las hipótesis auxiliares, de los instrumentos utilizados, de las observaciones realizadas, por un lado, y mediante la generación de hipótesis ad hoc que salven la teoría, por otro.

Así pues, Lakatos, p.ej., diría que lo que haría falta para que la revisión de la Teoría del Calentamiento Global fuese tomada en consideración no son evidencias empíricas, sino una teoría alternativa completa, que explicara todo lo que explica la teoría vigente y que fuera capaz de realizar mejores predicciones que esta. A lo que Kuhn añadiría que existen factores no estrictamente epistémicos que harían que la teoría se pudiera seguir sosteniendo incluso en este caso, puesto que el abandono de una teoría por otra es más una especie de "conversión religiosa" que el resultado de argumentos estrictamente racionales. Si esto fuera efectivamente así, los ejercicios de persuasión de los científicos serían más parecidos al proselitismo religioso o a la propaganda que a la argumentación racional basada en experimentos y el recurso a leyes y teorías previamente acreditadas. El debate sobre la TCG no quedaría entonces zanjado con argumentos sobre evidencias empíricas o sobre la consistencia con la Teoría del Efecto Invernadero. Ahora bien, si admitimos que ni los experimentos, ni el soporte de teorías acreditadas son argumentos suficientes para aceptar o rechazar un teoría científica ¿De qué se debate en las discusiones sobre teorías? ¿De qué depende que una teoría tenga éxito? Ciertamente, existen teorías de muy distinto tipo. Las teorías de la física y las teorías económicas, por ejemplo, son muy diferentes entre sí, y no es por lo tanto esperable una respuesta general, si lo que se pregunta es qué cosa en concreto se requiere para zanjar un debates científico. Ahora bien, sí es posible, sin embargo, proponer un esquema de análisis heurístico que sirva, en principio, para entender numerosos ejemplos. Una propuesta de este tipo es la que hacemos en este trabajo, ensayándola en el debate sobre la TCG. La elección de este debate se justifica en que el conocimiento que es posible tener de las discusiones entre colegas y de las acciones de los agentes externos es mucho mayor que en otros casos, debido precisamente a las filtraciones habidas, a las evaluaciones a las que estas han dado lugar, y a toda la información que se ha ido divulgando en los medios de comunicación. Insistimos en que el objeto de nuestra propuesta es la de comprender el funcionamiento de los debates científicos, no el de adjudicarlos, lo que es obviamente una tarea de los propios científicos, más que de los filósofos.

Para plantear la propuesta, partimos de un trabajo anterior (Zamora Bonilla 2006a, b) en el que se han presentado los elementos básicos de un enfoque inferencialista aplicado al análisis de la actividad científica. Este enfoque asimila la actividad de los científicos a un "juego de persuasión", en el que las jugadas son los argumentos utilizados, y el objetivo de cada jugador consiste en que los colegas, y en ciertos casos la sociedad en su conjunto, acepten las tesis, descubrimientos o propuestas defendidas por él. Este 
enfoque utiliza básicamente el aparato conceptual de la teoría de juegos de lenguaje y su característica fundamental, que lo distingue con respecto de la teoría económica de juegos de estrategia, es que el juego está sujeto a ciertas reglas de carácter normativo: no es la "utilidad" de los jugadores la principal explicación de sus movimientos, sino la existencia de normas que aquellos creen más o menos obligatorio obedecer. Según este enfoque, una comunidad científica está definida en parte por el conjunto de reglas de inferencia que se admiten en ella como válidas (es decir, qué esquemas argumentales se consideran admisibles). Cada miembro de la comunidad intenta resolver uno o varios "problemas" proponiendo alguna solución al mismo (aunque utilizamos los términos "problema" y "solución" en el sentido más general posible, abarcando desde problemas teóricos a cuestiones experimentales u observaciones de campo), y tiene que defender esa solución mediante argumentos que cumplan dos requisitos: 1) las premisas de esos argumentos deben ser aceptadas por el mayor número posible de los colegas, y 2) los esquemas argumentales utilizados deben ser considerados válidos por la comunidad. El uso de un esquema argumental $E$, que liga unas premisas $P$ con una conclusión $C$, significa que la comunidad considera que aquellos que están comprometidos con la verdad de $P$, tienen la obligación de aceptar $C$, salvo que propongan algún otro argumento considerado válido y que vaya en sentido opuesto. La tarea del científico consiste, por lo tanto, en intentar persuadir a sus colegas de que, dadas las reglas de inferencia que ellos aceptan, y dadas algunas de las tesis sobre su ámbito de estudio que ya aceptan, esos colegas deberían aceptar la solución que el primero propone. Naturalmente, como los esquemas argumentales aceptados no se reducen a las reglas de la lógica, y como las tesis previamente aceptadas pueden no ser del todo coherentes entre sí, el desarrollo de este juego puede llevar a que en una comunidad se acepten transitoriamente soluciones incompatibles a los mismos problemas, pero el trabajo de cada investigador consistirá, precisamente, en ir intentando acumular argumentos para que sus colegas acepten las tesis propuestas por él en vez de las de sus competidores. ${ }^{5}$

En el citado trabajo se proponía también un esquema de análisis evaluativo de la ciencia basado en dos distinciones (Zamora Bonilla 2006a, 196); primero la que distinguía entre la evaluación de las jugadas y la del juego propiamente dicho (es decir, entre los movimientos argumentativos concretos que realizan los participantes, por un lado, y las normas que la actividad argumentativa en su conjunto y la propia disciplina científica a la que dan lugar, por otro lado); y segundo, entre el punto de vista de los científicos participantes y los puntos de vista de los agentes "externos" (políticos, ciudadanos, medios de comunicación, empresas, agencias oficiales, etc.). Estas dos distinciones permiten establecer, pues, cuatro posibilidades: (1) análisis del debate entre colegas sobre argumentos concretos, que incluye la discusión sobre las fuentes de datos, sobre la evidencias empíricas, los métodos utilizados, y también incluye las disputas sobre si

\footnotetext{
${ }^{5}$ Nuestra propuesta tiene, naturalmente, muchos puntos en común con otros enfoques epistemológicos de tipo pragmático, tales como los de Laudan, van Fraassen, etc. No insistimos en las semejanzas con dichos enfoques porque el aspecto característico que deseamos resaltar es el aspecto competitivo del “juego de persuasión", es decir, aquello que permite interpretarlo como un "juego" en el sentido económico del término, y ese aspecto es, precisamente, el que, en nuestra opinión, no se ha estudiado suficientemente por parte de los enfoques anteriores.
} 
alguno de ellos está jugando mal; (2) la evaluación que los científicos hacen del conjunto de su campo científico, del juego como tal, y que responde a preguntas generales sobre si su proyecto es adecuado, si se está desarrollando eficientemente, o las oportunidades que ofrece para una buena carrera profesional; (3) la evaluación externa de los argumentos, que responde, por ejemplo, a cuestiones sobre la capacidad de los agentes externos para detectar las malas jugadas; y (4) la evaluación que los agentes externos hacen del conjunto de la disciplina. Es esta parte de nuestro enfoque la que queremos aprovechar expresamente para intentar arrojar algo de luz en el caso particular del debate sobre el cambio climático.

\section{La persuasión científica en el debate sobre la Teoría del Calentamiento Global. Análisis sistemáti- co mediante un enfoque inferencialista.}

\section{A. El debate entre colegas sobre argumentos concretos}

El debate sobre el gráfico de temperaturas es un ejemplo claro de discusión entre colegas de un argumento concreto. Aquí se trata, por un lado, de establecer que la relación entre el incremento de dióxido de carbono y el incremento de temperatura es una relación de causa a efecto., mientras que, por otro lado, se pone en duda esta relación cuestionando la correcta aplicación de los métodos estadísticos utilizados y la validez y fiabilidad de los datos

¿Qué datos se han considerado y cuales se han despreciado? ¿Es adecuado rechazar algunos datos? ¿Ha sido correcto el tratamiento estadístico? ¿Cómo se han suavizado las series temporales? ¿Qué filtros se han utilizado?, son discusiones que se cruzan con otras cuestiones como la acreditación para participar en el debate, la igualdad de oportunidades que los participantes tienen a la hora de exponer sus argumentos (controlando lo que se publica en las revistas especializadas, por ejemplo), la existencia de transparencia suficiente para poder valorar el trabajo de cada uno, y también la posibilidad de algún tipo de actuación deshonesta.

En el debate, estas discusiones no aparecen nítidamente separadas, pero pueden, no obstante, ser analizadas en distintos niveles, y pueden identificarse ciertas reglas y normas aceptadas explícita o tácitamente.

En un nivel preliminar ${ }^{6}$, se discute la admisión de alguien como interlocutor válido. Para ser admitido en un debate es necesario acreditar de algún modo un conjunto de conocimientos especializados. En el caso del que nos estamos ocupando, hace falta acreditar conocimientos suficientes sobre paleoclimatología; y, son necesarios también conocimientos sobre el tratamiento estadístico de datos. El círculo de científicos que pueden intervenir en el debate es por lo tanto reducido. Es algo similar a lo que sucede en una competición deportiva en la que se tienen que acreditar unas marcas mínimas para poder participar. Análogamente, para que las tesis de un investigador sean

\footnotetext{
${ }^{6}$ Existen otros niveles de discusión, sin duda interesantes, como por ejemplo, qué es una explicación científica y qué no lo es, qué tipo de relación tienen la teoría con la realidad, etc. Pero ese nivel tiene más importancia en la respuesta a la pregunta de qué discuten los filósofos de la ciencia, que en el análisis del desarrollo de una teoría científica.
} 
tomadas en serio, para que se considere oportuno dedicar un esfuerzo a rebatirlas o estudiarlas, se ha de recorrer un camino que pasa, por ejemplo, por participar en congresos o publicar artículos, lo cual aporta un reconocimiento de la capacidad para debatir. En este recorrido es a menudo importante contar con algún tipo de aval institucional. Algo similar a tener un buen entrenador o pertenecer a un club de prestigio.

Otro nivel de debate es el que se refiere a los conocimientos básicos de un cierto campo científico y el grado de consenso existente sobre su consistencia. De la aceptación de unos contenidos básicos se derivan una serie de implicaciones. Por ejemplo, la opinión favorable de un científico sobre el estado del arte de la dendrocronología obliga en principio a tener en cuenta los datos obtenidos con la metodología basada en ella. No se puede tener a la vez una buena opinión sobre esta rama de la ciencia y depreciar sin más los resultados obtenidos mediante ella cuando no corroboren la tesis que uno defiende.

Ser admitido como interlocutor válido, y que existan unos contenidos comunes, son, así, condiciones de posibilidad para que se pueda discutir sobre argumentos concretos. Esta discusión es la parte central del debate y las normas que operan aquí son principalmente normas metodológicas. El conjunto de metodologías puede ser muy amplio, pero contrariamente a lo que defendió en su momento Feyerabend, no todo vale. Al menos, dado un contexto específico, no todas las "jugadas" tienen el mismo valor para persuadir a los colegas de que se está argumentando de un modo válido y convincente.

En los correos filtrados, aparecen asimismo algunas cuestiones relacionadas con la calidad y honestidad de la producción científica. Se da a entender, por ejemplo, que algunas posiciones pueden ser vetadas en las revistas científicas, y que incluso una revista completa puede ser dejada fuera del círculo de la corriente principal, lo cual implicaría que posiciones consideradas poco ortodoxas podrían ver disminuidas sus posibilidades de argumentación y por lo tanto disminuida su fuerza de persuasión.

Finalmente, la discusión puede centrarse en las implicaciones que se derivan de la aceptación de una cierta argumentación. Por ejemplo, si algo se acepta como una anomalía hay que discutir si esta anomalía afecta o no a partes importantes de la teoría. Monbiot, por ejemplo, concluye en su artículo que la Teoría del Calentamiento Global está a salvo porque están a salvo las teorías de la física que le sirven de base. Pero en el supuesto, por ejemplo, de que la relación entre los anillos de los árboles y la temperatura estuviese suficientemente acreditada, y los anillos revelasen que hubo en la Edad Media temperaturas altas desvinculadas de la actividad industrial, significaría que el argumento principal que relaciona la actividad industrial humana con el calentamiento global perdería parte de su fuerza. Esta anomalía estaría atacando aspectos muy próximos al núcleo de la TCG, puesto que estaría cuestionado si el efecto invernadero está en la base del calentamiento actual de la atmósfera. Siguiendo el símil deportivo se ha de valorar si la presencia de la anomalía sirve simplemente para anotar un tanto en

\footnotetext{
${ }^{7}$ Metodología que relaciona características de tiempos pasados como la temperatura, basándose en el crecimiento de los anillos de los árboles
}

Theoria 76 (2013): 77-96 
el marcador de los escépticos, o si por el contrario sirve para decidir un partido, o incluso una liga.

La gran diferencia con las competiciones deportivas es que aquí no hay un arbitraje separado y tampoco hay un reglamento explícito, es decir, un conjunto de normas perfectamente definido. Los árbitros son los mismos participantes, o si se quiere, algunos participantes distinguidos, y el arbitraje es problemático tanto en la valoración de las implicaciones de los hechos de la experiencia como en la valoración de la correcta aplicación de los métodos científicos. Si bien no todo vale, la aplicación de los métodos tiene grados de libertad y es ahí, donde radica la necesidad de ser persuasivo. Por ejemplo, siguiendo con el caso del gráfico de temperaturas, hay distintos modos correctos de suavizar ${ }^{8}$ una serie estadística temporal, y por lo tanto pueden extraerse conclusiones diferentes, metodológicamente válidas, de unos mismos datos. En general, hay una parte del trabajo científico que es "artesanal" y, lo que es más importante, es dependiente de las hipótesis de partida del investigador, de la preconcepciones y teorías previas que tiene el científico sobre los fenómenos observados. Un dato que para un científico es necesariamente anómalo, para otro es la confirmación empírica de que sus sospechas iban por buen camino. Por ello, aun en los argumentos concretos, se trata más de persuadir que de demostrar que se está en lo cierto. Se trata de convencer de que, dentro de los grados de libertad existentes, se han hecho las elecciones del modo más racionalmente justificable y con el mayor rendimiento explicativo posible.

\section{B. Evaluación interna del conjunto del campo científico}

Un campo prometedor es aquel en el que se son esperables resultados teóricos relevantes para la sociedad y reconocibles para los científicos que trabajen en ellos. En algún momento, cada científico sopesa, por ejemplo, la oportunidad de formar parte del grupo que cree en una línea de investigación, o de colocarse entre los que se consideran escépticos con respecto a los resultados que se van obteniendo, en función de lo que esto puede contribuir a su carrera científica. Ciertamente, para que una investigación como la que requiere la ciencia del cambio climático vaya adelante, hace falta que participe en ella un número mínimo de científicos, lo cual significa que algunos científicos que potencialmente pueden ser atraídos hacia otros campos, trabajen en este, y en algún momento tienen que ser persuadidos para hacerlo.

La capacidad de atracción del campo tiene que ver también con las condiciones de eficiencia y equilibrio existentes. En el caso de la investigación sobre el clima, los recursos dedicados han sido y siguen siendo enormes, y también lo son las incertidumbres remanentes. La capacidad para mostrar que los fondos han sido empleados de forma eficiente en el pasado y que serán bien empleados en el futuro es pues relevante.

\footnotetext{
${ }^{8}$ Suavizar o filtrar una serie estadística temporal consiste en determinar la tendencia de la serie, es decir, el cambio de los valores medios. Lo que se pretende al suavizarla es eliminar, por ejemplo, la estacionalidad o los efectos aleatorios.
} 
Para ser atractivo, el campo ha de tener además unas ciertas condiciones de equilibrio y ha de preverse una cierta estabilidad en un tiempo razonable, ya que las grandes inercias asociadas a la formación de los investigadores y a la creación de equipos no permiten la adaptación inmediata a nuevas situaciones.

En la investigación del clima, la necesidad de obtener datos de fuera del propio país siempre fue sentida como imperiosa, y por ello las organizaciones meteorológicas fueron pioneras en la convocatoria de congresos internacionales. Unos pocos científicos, con alguna intervención de ciertos diplomáticos, fueron capaces de organizar, por ejemplo, el Año Polar de 1932.

Después de la Segunda Guerra Mundial, esta tradición se recogió como un precedente para convocar eventos de este tipo entre científicos preocupados por lograr que las investigaciones fueran más allá de los intereses de los gobiernos nacionales, que son considerados un serio obstáculo para la eficiencia de las investigaciones. Así, en 1951 se crea la $\mathrm{OMM}^{9}$ que pronto se convierte en una agencia de las Naciones Unidas. Desde estos foros internacionales se trata de persuadir a los gobiernos de la necesidad de realizar grandes inversiones para mejorar el conocimiento del planeta y en particular del clima; con cierto éxito, puesto que durante el Año Geofísico Internacional de 1957, la existencia de fondos permite que se midan los niveles de dióxido de carbono, para descubrir que están subiendo, a pesar de que el cambio climático no es en ese momento una prioridad.

En 1961, los esfuerzos de los científicos van dirigidos a aumentar las inversiones con objeto de mejorar los sistemas de medición, encontrando un aliado: los planes espaciales del presidente Kennedy. El lanzamiento de satélites al espacio sirve muy pronto para tomar datos sobre el clima. Durante los años siguientes, aunque las dificultades para vencer las inercias nacionales permanecen, algunos científicos son capaces de crear comités internacionales que tienen continuidad más allá de los pocos días que duran los congresos, y se establece una sede permanente en Ginebra. En este marco, los encuentros son más frecuentes y comienza a formar parte de las agendas el cambio del clima inducido por los contaminantes.

En 1971, algunos científicos llegan a ser tan convincentes que logran que el tema del impacto humano sobre el clima sea de lectura obligada en la reunión preparatoria de la Conferencia de Estocolmo. Durante los años siguientes continúan los esfuerzos individuales. Por ejemplo, Robert M. White ${ }^{10}$, lo hace desde su puesto en la oficina meteorológica de los EEUU y en la OMM. En 1979 el resultado de este y otros esfuerzos es la convocatoria de la Conferencia Mundial sobre el Clima. En 1981, el fruto de los trabajos que divulgan las posibles catástrofes vinculadas con el cambio climático, anunciadas por ejemplo por S. Schneider (1976) o J. Hansen, este último desde la NASA, comienza a recogerse en los grandes medios de comunicación. Por ejemplo, siguiendo el consejo del escritor científico W. Sullivan, los editores del New York. Times llevan a la primera página el tema del calentamiento global.

\footnotetext{
${ }^{9}$ Organización Meteorológica Mundial (WMO en inglés).

${ }^{10}$ Spencer Weart (2004) ha estudiado estos detalles.

Theoria 76 (2013): 77-96
} 
En los años ochenta, se configura una especie de comunidad internacional del clima, que en la Conferencia de Villach de octubre de 1985, está madura. Lo que une a esta comunidad es el convencimiento de que el cambio del clima es más rápido y más intenso de lo previsto. A pesar de no presentarse nuevos desarrollos espectaculares, en distintas reuniones y foros se sacan conclusiones favorables a la implementación de medidas para mitigar el cambio climático, algo que se ha explicado ${ }^{11}$ por el decisivo apoyo de las agencias internacionales, influidas a su vez por una comunidad científica internacional bastante compacta, liberada ya de las restricciones nacionales impuestas en la posguerra. A partir de 1985, las actividades para convencer de la amenaza del cambio climático se hacen constantes y se encuentran nuevos aliados, algunos no buscados. Por ejemplo, aquellos que ven en la lucha contra el cambio climático una oportunidad para la regulación, pero también, aquellos que ven en este asunto un discurso político ambientalista en el que explayarse, por citar dos casos muy diferentes.

A todo esto, se une la preocupación por la capa de ozono que da como resultado el Protocolo de Montreal. Un acuerdo que es recibido como un éxito y que anima la convocatoria de la Conferencia de Toronto de 1988. La comunidad científica que se reúne en Toronto es básicamente la que se había formado en Villach y su posición es por lo tanto prácticamente unánime con respecto a las causas y efectos del cambio climático. Ese mismo año, y promovido por la OMM y el PNUMA ${ }^{12}$, ambos organismos insertados en la organización de las Naciones Unidas, se constituye el Panel Intergubernamental para el Cambio Climático (IPCC ${ }^{13}$ ) con la vocación de ser un organismo que trascienda lo meramente científico y que sirva como consejo científico de las políticas de los gobiernos.

La historia que ha llevado a la constitución del IPCC va ligada al desarrollo de la TCG. Las coyunturas favorables o desfavorables y las confluencias de intereses han ido impulsando u obstaculizando la investigación y no han sido en absoluto irrelevantes. Como tampoco es irrelevante el hecho de que las comunidades científicas estén formadas por personas que tienen trayectorias vitales concretas. Los científicos juegan habitualmente en equipo formando parte de instituciones científicas con formaciones tenidas en común en las que se comparten metodologías, concepciones y formas de hacer las cosas. No es indiferente cuál ha sido el grupo que se ha convertido en dominante dentro del IPCC para que la teoría se haya desarrollado por el camino en el que lo ha hecho.

Por otra parte, dentro de los equipos y dentro de las instituciones, hay jugadores destacados. Ciertos científicos individuales tienen un papel más influyente que otros. Efectivamente, en el desarrollo de la teoría ha influido el esfuerzo de persuasión realizado por algunos científicos individuales. Han existido esfuerzos muy notables dedicados a convencer de la relevancia del problema del cambio climático, que a veces han tenido un carácter casi misional, compatible por otra parte, con la percepción del campo como prometedor para el desarrollo de una carrera científica.

\footnotetext{
11 "The development of an agenda for climate change: connecting science to policy" (Franz 2007).

${ }^{12}$ Programa de las Naciones Unidas para el Medio Ambiente.

${ }^{13}$ Se utiliza normalmente el acrónimo de la denominación en inglés.
} 
Las instituciones internacionales son precisamente un foro que permite que algunos investigadores individuales se conviertan en protagonistas de mensajes que son asumidos por el conjunto de la comunidad científica reunida en ellos. Para alcanzar esta capacidad de influencia es necesario en cualquier caso un fuerte poder de convicción, que procede en algunos casos del sentimiento de que se cumple con una misión. De algún modo, en la propia creación del IPCC ha tenido mucho que ver el convencimiento de algunos científicos de que estaban en la obligación de salvar al planeta de la contaminación producida por los combustibles fósiles. En el relato del origen y desarrollo del la Teoría del Calentamiento Global (en el que ha trabajado especialmente $\mathrm{S}$. Weart) puede identificarse a algunos científicos ${ }^{14}$ proclives al activismo ${ }^{15}$, que a pesar de las incertidumbres existentes en el conocimiento del problema van viendo claros signos que avisan del impacto destructivo que el hombre está teniendo sobre el planeta. Desde este convencimiento, consideran justificado construir historias impactantes que interesen a los medios y a la opinión pública, aunque el contenido científico de estas historias sea escaso ${ }^{16}$, como un modo legítimo de persuadir a los políticos y a la opinión pública de la importancia del problema. Esta estrategia ha resultado históricamente eficaz. Es un hecho, por ejemplo, que la conferencia celebrada en Toronto en junio de 1988 atrajo tal cantidad de periodistas que hubo que habilitar nuevos recintos para la prensa, no previstos, con objeto de alojar a las hordas de periodistas que acudieron al evento, y también es cierto que una encuesta realizada en 1989 reveló que el porcentaje de americanos que había oído hablar del efecto invernadero había pasado del 38\% de 1981 al $79 \%$ de ese momento.

En la ciencia del cambio climático existen además otros temas relevantes, que aun referidos a cuestiones metodológicas, son suficientemente generales para ser tratados desde el punto de vista del conjunto del campo. Por ejemplo, algunos científicos (Betz 2009) están cuestionando hasta qué punto algunos modelos matemáticos se están utilizando correctamente o no en los informes generales del IPCC. Según este punto de vista, los modelos que se utilizan en climatología adolecen de un problema general de infradeterminación, es decir, no son capaces de dar resultados concluyentes, solamente resultados que pueden ser posiblemente ciertos. La incertidumbre, dicen, es inherente a los modelos que tratan de representar el comportamiento del clima y no es posible ni siquiera asignar probabilidades a los escenarios futuros. Un desacuerdo sobre la forma de tratar la incertidumbre puede convertir los debates sobre argumentos concretos en diálogos de sordos, y en este sentido esto es algo que ya hemos visto en al apartado anterior, pero desde un punto de vista más sociológico, estos desacuerdos pueden producir divisiones dentro del campo, que si no son resueltas pueden llevar a inefi-

\footnotetext{
${ }^{14}$ James E. Hansen ante el Iowa Utilities Board comparó los trenes cargados con carbón, con los "trenes de la muerte" (A. Revkin se refiere a este comentario en un artículo del New York Times del 26/11/2007: "Climate, Coal and Crematoria").

${ }^{15}$ T. Gilland se refiere a esta cuestión en un ensayo escrito en junio de 2007: "Digging the roots of the IPCC" (Gilland 2007).

${ }^{16}$ Gilland cita a Schneider en su afirmación de que algunas historias tienen "muy poco contenido científico".
} 
ciencias y a desequilibrios, en el sentido de que algunos científicos tienen que ir reubicándose dentro del campo a medida que son o no persuadidos de la relevancia del problema. En estos reajustes se pierde o gana reconocimiento dentro del campo y son esperables defensas tenaces de las posiciones de cada uno con objeto de mantener el status.

Asimismo, la correcta utilización de los modelos coste/riesgo/beneficio, es un problema que ha generado una ingente producción de literatura científica, de lo que son ejemplo claro las discusiones relacionadas con el "Informe Stern". Este informe incluye argumentos multidiscipliares que integran economía, clima y medio ambiente. Lo relevante en este caso es el tipo de conexión que se establece entre los distintos campos. Cada campo tiene metodologías propias y la evolución del modelo conjunto, y por lo tanto la evolución del desarrollo conjunto de las teorías que intervienen, dependerá de qué campo sea el dominante.

De acuerdo el planteamiento que estamos siguiendo cabe preguntarse también si existe algún tipo de normas que regulen estos problemas de carácter más general. En el caso de la ciencia del cambio climático la respuesta es afirmativa. La comunidad internacional, ha establecido ${ }^{17}$ que en la investigación del clima ha de cumplirse el Principio de Precaución, de acuerdo con el cual debe ser investigado todo aquello que pueda desembocar en riesgos gravísimos para la humanidad, aunque sea poco probable. Esta norma, mientras esté vigente, condiciona la investigación, aunque, naturalmente, ella misma, o su "apropiada" interpretación, puede convertirse y se convierte a menudo en objeto de debate. Pues todo lo posible entra a formar parte del objeto a investigar, y una discusión que se vuelve entonces central es si la norma misma es adecuada, en qué foro se ha de discutir esto y quienes han de decidirlo.

\section{Evaluación externa de los argumentos cientificos}

El control social de la actividad de los científicos está siendo percibido como una necesidad creciente. Prueba de ello es la proliferación de organismos que tratan de un modo u otro de garantizar o controlar la calidad de la producción científica.

La evaluación externa de los argumentos científicos se está planteando, en la práctica, en distintos niveles. En un primer nivel, se está realizando una evaluación muy próxima a la que se hace entre colegas, mediante comités de expertos que trabajan por encargo para dilucidar asuntos concretos. En otro nivel, algunas administraciones nacionales están tratando de evaluar cuestiones relacionadas con la deshonestidad científica a través de comités ad hoc constituidos por expertos en el tema científico que se evalúa, pero con miembros que conocen de la legislación vigente, como es el caso del Comité de Deshonestidad Científica danés, por ejemplo.

El listado de malas prácticas que ha establecido este comité incluye: la ocultación del modo en que se han elaborado los datos, el hecho de deshacerse de forma selectiva de algún resultado, o el hecho de ocultar el uso poco habitual y erróneo de métodos estadísticos.

\footnotetext{
${ }^{17}$ Convención Marco sobre el Cambio Climático. Aprobada en 1992, entra en vigor en marzo de 1994.
} 
Lo cierto es que este tipo de evaluación externa esté siendo cuestionado. En algunos artículos (Rörsch 2008) aparecidos a raíz del caso Lomborg ${ }^{18}$ se ha defendido que la evaluación externa quede confinada a la aplicación de normas y criterios meramente éticos de carácter general, como el plagio o la transparencia, por ejemplo, sin entrar en los detalles de los argumentos científicos utilizados. Se trataría entonces de una evaluación más próxima a la de los tribunales de justicia, que basa sus veredictos en el derecho positivo: La Ley de Derecho a la Información o la Ley de Protección de Datos, por ejemplo. La cuestión clave es si los agentes "externos" pueden detectar las malas prácticas científicas y con ello intervenir en el desarrollo de una teoría científica.

El caso de los correos filtrados ha proporcionado en el año 2010 nuevos datos sobre el trabajo de los comités externos, que permiten abordar esta cuestión con mayor profundidad. Durante el año 2010, se han ido conociendo los informes de tres comités de investigación. Uno de los comités depende del parlamento británico, mientras que los otros dos son el resultado de sendos encargos de la universidad de East Anglia.

El parlamento británico ha realizado su investigación a través del Comité de Ciencia y Tecnología ${ }^{19}$, que se ha centrado en varias cuestiones: la transparencia, la honestidad del trabajo realizado en la CRU, la posible perversión del proceso de revisión entre colegas y la propia Ley de Libertad de Información. En la investigación sobre la posible actuación deshonesta de los científicos de la CRU, el comité no tiene ninguna duda de que el correo filtrado que causa más preocupación es el que habla del "truco $[\ldots]$ para esconder el descenso". Sobre la palabra "truco", el comité recaba distintas interpretaciones. Alguna de ellas se refiere a que el truco no es ni más ni menos que la falsificación de datos; no obstante, las explicaciones de la CRU se refieren a esta como una expresión coloquial que significa "la mejor forma de hacer algo". El comité haciendo un balance de las evidencias disponibles admite esta interpretación y admite también que la CRU simplemente ha despreciado datos que pensaba honestamente que eran erróneos.

En el tema de la posible distorsión del procedimiento de revisión entre colegas que se derivaría del abuso realizado al impedir que se publiquen puntos de vista diferentes a los de la corriente principal, el comité conviene en que los científicos no deben ser criticados por hacer simples comentarios informales.

Los asuntos relacionados con la Ley de Libertad de Información son tratados también extensamente. La ley, afirma el comité, crea nuevos derechos de acceso a la información que afectan a todos aquellos que reciben fondos públicos. En caso de conflicto, es más importante la transparencia que la protección de datos cuando estos dos derechos colisionan. Sólo han de aplicarse excepciones cuando el interés público en proteger datos sea superior al interés que la opinión pública tiene en conocerlos. Es el inte-

\footnotetext{
${ }^{18}$ B. Lomborg, autor de El ecologista escéptico (2001), fue acusado ante el Comité danés, de mala práctica científica. El comité falló inicialmente en contra de Lomborg, pero el fallo fue recurrido ante el Ministerio de Ciencia, del que dependía, que no consideró demostrada la mala práctica.

19 "The science and Technology Commitee" que depende de la cámara baja británica (The House of Commons). Fue creado el 1 de octubre de 2009 con los mismos miembros que el anterior Comité de Innovación, Universidades y Ciencia (Innovation, Universtities, Science, and Skills Committee).
} 
rés público el que debe prevalecer. Y a la luz de estos criterios es como deben juzgarse cada actuación concreta.

El comité, tras el estudio de los correos, detecta que en la CRU permanece una cultura que no favorece la apertura, reteniendo información a aquellos que se consideran hostiles a la tesis del calentamiento global y que esto es inaceptable. Concluye que la Universidad de East Anglia ha permitido este comportamiento y que necesita por lo tanto revisar su política en este punto.

La Universidad de East Anglia en diciembre de 2009 abrió dos investigaciones ${ }^{20}$ : una sobre el asunto mismo de los correos filtrados, y la otra es una evaluación científica general del trabajo de la CRU. En julio de 2010 se publica el informe del comité independiente que revisa las posibles malas prácticas que parecen deducirse de los correos filtrados. Sobre los registros de temperaturas procedentes de estaciones terrestres, el comité concluye que la CRU no ha retenido datos y que tampoco ha habido sesgo en la selección de las estaciones, pero que sin embargo la CRU debería haber sido menos ambigua y estar menos a la defensiva a la hora de facilitar el listado de estaciones utilizadas, aunque que esto no mina la confianza en el trabajo global de la CRU. Sobre el discutido gráfico de temperaturas el comité concluye que no es desorientador en sí mismo pero que tenían que haberse explicado más claramente los procedimientos utilizados en la reconstrucción de datos, bien en el título o bien en el texto. El comité considera que no ha habido mala conducta en el proceso de revisión entre colegas ni en la presentación del gráfico al público ni a los gestores políticos, y una vez más, se hace recaer sobre la Universidad de Easta Anglia, como institución, la falta de diligencia en las respuestas a los requerimientos de la FOIA.

A pesar de todo ello, en el apartado de asuntos de más alcance, el comité considera escaso el uso que la CRU ha hecho de la metodología científica basada en la búsqueda y verificación, o en su caso falsación, de hipótesis alternativas, que las incertidumbres asociadas a cada alternativa deben ser manifestadas y que a los gestores políticos se les deben mostrar los puntos de vista alternativos. El comité considera que la revisión entre colegas es una pieza clave del progreso científico, pero que no debe exagerarse su validez en piezas singulares de investigación y que no es bueno impedir que se publiquen resultados de investigaciones contrarios a la corriente principal.

Precisamente porque existen normas es posible vulnerarlas y es posible entonces la deshonestidad científica. Es posible la falsificación y la ocultación de datos, así como la utilización sesgada de herramientas estadísticas. Ahora bien, no puede concluirse que la deshonestidad científica sea una práctica extendida. En los casos evaluados, las cuestiones denunciadas resultan ser finalmente discutibles y no se han detectado casos flagrantes de deshonestidad científica. Por lo tanto, la mala práctica intencionada no tiene un peso determinante en el éxito o fracaso de la teoría. Es decir, no puede afirmarse que la teoría haya tenido éxito, porque los que la han defendido han actuado de

20 "The independent climate change e-mails review", dirigido por sir Muir Russell, da como resultado un informe de fecha 7 de Julio de 2010, al que se puede acceder en http://www.cce-review.org. El "Scientific Appraisal Panel", dirigido por Lord Oxburgh, da como resultado un informe que se remite a la Universidad de East Anglia el 12 de abril de 2010, al que se puede acceder en http://www.uea.ac.uk. 
forma deshonesta. Esto no significa que la ciencia esté libre de errores, ni que la preocupación por la calidad de la producción científica no sea una preocupación plenamente justificada. La distorsión de la revisión entre colegas existe. Aunque no actúen de mala fe, en la práctica, algunos científicos son capaces de colocar barreras frente a la divulgación de posiciones científicas que consideran equivocadas u hostiles a la suya propia. Permanece asimismo una cultura de cierta ocultación, de falta de transparencia. Los comités investigadores han detectado un cierto comportamiento atávico, que mantiene costumbres que fueron la norma en otras épocas. Durante siglos, el ocultamiento fue considerado un valor para la ciencia, puesto que el conocimiento debía permanecer dentro del círculo de aquellos que eran capaces de comprenderlo y utilizarlo adecuadamente. Esta mentalidad no forma parte de los principios éticos de las sociedades democráticas contempóraneas y la legislación que regula la transparencia de la actividad científica tiende a aumentar, y esto es algo con lo que tiene que contar los científicos y que condiciona el modo en que se desarrollan los debates.

\section{Evaluación externa del conjunto de la actividad de los científicos del clima}

Las grandes compañías petrolíferas se interesaron muy pronto por las posibles implicaciones que sobre su actividad industrial podría tener lo que se iba sabiendo acerca de la relación entre emisiones de dióxido de carbono y cambio del clima. En el año 1980, antes de que se constituyera el IPCC, la compañía Exxon ${ }^{21}$ fue pionera en la investigación sobre el cambio climático. Contrataron para ello los servicios de un astrofísico de Harvard, Brian Flannery, para tratar de aplicar modelos matemáticos al problema del calentamiento global. En los años siguientes, se unieron al equipo investigador más expertos $^{22}$, y cuando se constituye el IPCC y emite sus primeros informes, la compañía se siente con información propia suficiente para afirmar ${ }^{23}$ que las incertidumbres científicas existentes aconsejan no tomar decisiones tecnológicas precipitadas.

Realizar sus propias investigaciones no es el único movimiento estratégico que protagoniza la Exxon. Junto con otras compañías, en 1989 funda la Global Climate Coalition (GCC), un grupo que se constituye para dar respuesta a los informes del IPCC. El grupo funciona con bastante intensidad como lobby durante la década de los noventa, hasta el año 2001, año en que algunas empresas importantes se desmarcan coincidiendo con la publicación del tercer informe del IPCC. Estas acciones son reiteradamente denunciadas por los medios de comunicación y las organizaciones favorables a la reducción de emisiones. Con todo, la conducta que genera más polémica es la financiación de grupos de investigación externos a la compañía, algunos con sede en universidades (e.g., Stanford) a los que se acusa de científicamente desorientadores. Con esta labor de financiación han sido especialmente duros la Union of Concerned Scientists, la Royal Society (especialmente a través de su portavoz, Bob Ward) y los grupos

\footnotetext{
${ }^{21}$ Standard Oil Company of New Jersey. A partir de 1999, junto con la Standard Oil Company of New York forman la Exxon Mobil.

${ }^{22}$ En 1987 se une Haroon Kesgui procedente del Lawrence Nacional Laboratory de California.

${ }^{23}$ El portavoz principal de la Exxon hasta el año 2005 fue el CEO de la compañía, Lee Raymond
} 
ecologistas. El caso es que a partir del año 2007 se detecta un cambio de estrategia en la compañía, moderando su posición. El cambio responde a un nuevo estado de cosas: en primer lugar, los informes del IPCC se van haciendo más consistentes y menos vulnerables a la crítica; en segundo lugar, algunas compañías importantes se van alejando de la oposición radical a la reducción de emisiones y consideran la opción de adoptar tecnologías basadas en energías renovables; en tercer lugar, sobre todo a partir del año 2005, van apareciendo informaciones sobre la llegada próxima del cenit del petróleo que aconsejan la transferencia hacia tecnologías no basadas en combustibles fósiles cuyas reservas son finitas y que posiblemente se agotarán en un tiempo no muy lejano. La posición inicial era por otra parte justificable, desde el punto de vista de la industria del carbón y del petróleo, si se tienen en cuenta las grandes inercias que tienen las inversiones tecnológicas en este tipo de actividad, puesto que realizada la apuesta estratégica de invertir en un determinado desarrollo tecnológico, se requieren bastantes años para dar un giro hacia otro distinto.

En este contexto se produjo uno de los episodios (vid. nota 14) cuyo análisis resulta más interesante. En al año 2001, el IPCC estaba dirigido por Robert T. Watson, al que la industria del carbón y del petróleo calificaba de firme defensor de la reducción de emisiones, una reducción propuesta por el Protocolo de Kyoto. Ese mismo año, llega a la presidencia de los Estados Unidos G. W. Bush y la industria logra persuadir a la entrante administración de que los resultados científicos que están saliendo del IPCC son altamente dañinos para los intereses del país. Se considera necesario que la dirección del IPCC sea ocupada por otra persona que, desde su punto de vista, no sesgue los resultados de los informes a favor de la reducción de emisiones. Se realizan entonces una serie de movimientos para que Watson no salga reelegido. El caso es que en abril de 2002, efectivamente, Watson no sale reelegido y la dirección del IPCC recae en R. Pachauri2 ${ }^{24}$ que parece contar con el visto bueno de la Administración de los EEUU. Pachauri que ocupaba hasta entonces la vicepresidencia, es economista y como tal se le supone más sensible a las repercusiones económicas de las medidas contra el cambio climático. Pero lo más interesante del asunto es que la nueva dirección apenas produce cambios sustanciales en los informes que genera el IPCC, que tienen una clara continuidad con los anteriores.

Ciertamente, la voluntad de la industria de que se sustituya la dirección del IPCC tiene que pasar por la persuasión a la administración, y la administración de los EEUU tiene a su vez que plantear la sustitución ajustándose a las normas estatutarias del IPCC y convencer al número de países que hacen falta para ganar la elección. Tampoco es posible presentar a cualquier candidato, sino que hay que tratar de buscar a alguien que cumpla con el perfil adecuado, y de hecho, la persona que se presenta es en ese momento vicepresidente. Finalmente, y más importante, el IPCC tiene unas normas de funcionamiento interno que hacen que los resultados sean relativamente independientes de la persona que lo dirige. Después de doce años de funcionamiento, la nueva presidencia encuentra ciertas inercias en las que cada escalón del sistema va haciendo lo que se supone que tiene que hacer y por ello los resultados tienen continui-

${ }^{24} 75$ países votan a favor de R. Pachauri, 49 a favor de Watson. 
dad con el trabajo anterior. Puede decirse que en ese momento El IPCC funciona ya con cierta autonomía, tiene sus propias normas. La acción de intentar intervenir sobre los informes que resultan del trabajo científico del IPCC se encuentra con una maquinaria en funcionamiento que reacciona ante ese intento. El nuevo director del IPCC habría actuado, según esta interpretación, haciendo lo que creía que tenía que hacer en función de su recién adquirida responsabilidad y del funcionamiento de la organización.

Por otra parte, el trabajo de los científicos del clima, y en concreto, el trabajo del IPCC, está siendo valorado también, como es lógico, por el público y los medios de comunicación, aunque el análisis de estas evaluaciones requeriría un trabajo al menos tan largo como el presente, de modo que preferimos dejarlo para una ocasión posterior. ${ }^{25}$ De las numerosas ramificaciones que tiene este tema, interesa destacar, desde el punto de vista del enfoque que estamos siguiendo, que los medios son a menudo el terreno de juego en el que se producen las disputas más encendidas. Las posiciones de los colegas se conocen a veces a través de los medios. Esto implica, por un lado, que si se quiere ser persuasivo se tiene que dominar el estilo que exigen los medios; y por otro lado, que ese estilo tiene peculiaridades como la simplificación, la exageración o la presentación indiscriminada de la información. En definitiva el debate en los medios se rige por unas normas específicas. Por ejemplo, un ensayo escrito para un congreso tiene un formato distinto al de un artículo escrito para una revista general. Tienen ventaja en el juego aquellos que puedan y sepan servirse de ellas. Cuestión distinta es si esta ventaja es sostenible a la larga, o bien los argumentos más racionalmente justificables se abren finalmente camino de modo necesario.

En el proceso de elección de teorías influyen las preferencias de los sectores de la sociedad que valoran el trabajo de los científicos. Hay que analizar pues estas preferencias si se quiere tener una visión más amplia del modo en que esta elección se produce. La inclinación hacia la teoría del efecto invernadero, que coloca como responsable del cambio climático a los combustibles fósiles, no es ajena a la preocupación por la conservación del medio ambiente, presente en sectores muy activos de la sociedad que se genera en los años sesenta y setenta. Existen tanto alicientes como elementos de disuasión externos, que alientan o retraen una investigación. Los recursos económicos pueden ser muy condicionantes en el caso de investigaciones que requieran experimentos costosos, pero las oportunidades para el reconocimiento pueden operar como un aliciente mayor.

Las preferencias de los agentes externos actúan a veces de un modo sutil, pero cabe esperar que los intereses de los agentes puedan actuar en ocasiones de un modo más compulsivo. Son lugares comunes las referencias a los intentos de la industria y de la Administración para orientar o desorientar las investigaciones sobre el cambio climático, pero los científicos han demostrado, con la cobertura de las instituciones, tener una capacidad de resistencia notable frente a las presiones externas. Por otra parte, Los medios de comunicación tienen una grandísima influencia en la divulgación de

${ }^{25}$ Veáse, por ejemplo, Gavin, Leonard-Milson \& Montgomery (2009); Zia \& Todd (2010).

Theoria 76 (2013): 77-96 
teorías, y en la percepción pública de qué teoría es una buena teoría, pero no está tan claro que puedan orientar el trabajo de los científicos.

\section{Resumen y conclusiones}

Las categorías que hemos utilizado tienen ciertamente contornos borrosos. Así, la propia acotación del campo científico es problemática porque todos los campos están en última instancia interconectados; también lo es la distinción entre los argumentos concretos y las discusiones que se refieren a las normas vigentes en el conjunto del campo; y asimismo, los científicos no está separados nítidamente del resto de actores. Sin embargo, a pesar de ello, el enfoque utilizado se ha mostrado útil para sistematizar el análisis y dar sentido a un amplio conjunto de hechos que pueden ser interpretados como formando parte de un juego de persuasión regulado por ciertas normas.

En el caso estudiado, el campo científico es la ciencia del clima y el juego consiste en que cada jugador trata de que se acepten sus tesis y descubrimientos como la mejor explicación de ciertos fenómenos observados, cuya relevancia y alcance también se discute. Los agentes externos, que consideramos arbitrariamente como todos aquellos que no pertenecen a la comunidad científica del campo, son observadores activos, observadores participantes que influyen el desarrollo del juego. Las jugadas básicas son los distintos argumentos que se utilizan en la defensa o ataque de las tesis, pero no todas las jugadas son de este tipo; p. ej., vetar la presentación de una ponencia o retirar los fondos de una investigación son acciones que también pueden influir en el desarrollo de una teoría.

La propuesta realizada en este trabajo contiene dos presuposiciones básicas. La primera, que la actividad de los científicos es asimilable a un juego de persuasión; y la segunda, que en este juego no todo vale sino que está sujeto a ciertas normas. Después de analizar el caso, estamos en mejores condiciones para explicar qué es lo que hace que la actividad de los científicos pueda ser interpretada como un juego de persuasión y qué es lo que la distingue, por un lado, de un ejercicio continuado de demostración; es decir, de un conjunto de razonamientos que llevan, desde algo evidente o ya demostrado, a lo nuevo que se quiere afirmar; y por otro lado, que la distingue de un simple ejercicio de propaganda en el que se van ganando adeptos sin importar cómo.

La persuasión es necesaria porque hay elecciones a cada paso y porque no hay unas alternativas mejores que otras de un modo absoluto, sino que hay distintas maneras aceptables de hacer las cosas. Es fácil admitir que lo que se investiga y con qué intensidad se hace depende de hechos circunstanciales que despiertan el interés por un determinado aspecto del mundo. Pero también está condicionado por la capacidad de persuasión de individuos, grupos e instituciones, que tienen percepciones personales o colectivas de la relevancia de un problema. Una consecuencia importante es que la teoría se desarrolla de un modo distinto dependiendo de qué individuos, grupos o instituciones sean capaces de influir de un modo más efectivo. Al contrario de lo que pudiera parecer, la mayor capacidad de influencia no reside en las instituciones o grupos que tienen más poder en otros órdenes, poder económico o político, por ejemplo. Científicos individuales o la comunidad científica de un campo, tienen una notable capacidad de resistencia frente a influencias externas. Por ello, el camino que sigue el 
desarrollo de una teoría depende sobre todo de los científicos del campo, y es en las peculiaridades del campo y su relación con otros campos donde hay poner el foco de atención para entender la evolución de la teoría. En la caracterización del campo, hay que atender, tanto a aspectos epistemológicos, como por ejemplo los métodos que se utilizan preferentemente en el campo, como a los aspectos sociológicos, tales como la capacidad de atracción de un cierto campo científico para el desarrollo de una carrera profesional.

Pero incluso en los argumentos concretos, la persuasión ejerce un papel importante. En este trabajo hemos dibujado solamente los trazos más gruesos de las elecciones que hay que hacer a cada paso de una argumentación concreta. Hemos tratado de destacar que en la presentación del gráfico de temperaturas, por ejemplo, hay que decidir qué datos son relevantes y qué tratamiento se da a esos datos; y más importante, que estas decisiones tienen distintas alternativas razonables, tienen grados de libertad, por lo que hay que persuadir a los que hacen elecciones diferentes de que la elección realizada es la mejor.

Por otra parte, persuadir no es engañar. La deshonestidad científica es posible y se puede dar un listado de prácticas deshonestas, pero no parece que sea una práctica extendida. En los casos evaluados, finalmente, parece que los científicos hacen lo que creen que tienen que hacer. El control de los colegas resulta ser bastante efectivo y una práctica deshonesta descubierta acabaría con el reconocimiento de un científico.

A veces, la defensa de un argumento, o de un conjunto de argumentos, es asimilable a la defensa que se hace en un juicio, delante de un tribunal. Se produce esto cuando se defienden las tesis delante de un comité. La efectividad de estos comités depende por supuesto de la capacidad de los expertos que los forman, pero depende también del alcance de la evaluación. Cuando se juzgan argumentos concretos, no pueden ir más allá de la revisión entre colegas, de la que son completamente dependientes. Sin embargo, pueden ser efectivos en la valoración de algunas cuestiones importantes; la transparencia o la propia distorsión de la revisión entre colegas son dos de ellas.

La tenacidad es una gran virtud de los científicos, que les permite resistir presiones externas e impulsar investigaciones, pero esta virtud puede ser a la vez su mayor vicio. Los científicos tienen una gran tendencia a sostener sus hipótesis. Esto es de hecho potencialmente la principal fuente de errores que denuncian los comités que se ha formado a raíz del caso de los corre filtrados. Estos errores se derivarían se derivarían de que la búsqueda y valoración de distintas hipótesis alternativas no es la práctica metodológica habitual. En el vocabulario de la filosofía de la ciencia puede decirse que las hipótesis se convierten en una carga teórica que pesa sobre el conjunto de la investigación; no sólo sobre la fase de observación, sino en todo el proceso investigador. Los científicos son excesivamente tenaces en la defensa de la hipótesis elegida y no están lo suficientemente abiertos a la consideración de hipótesis alternativas, menos cuanto más avanzada está la investigación.

El lado más racional de la ciencia es la existencia de normas que regulan los procedimientos aceptados. Cómo se generan estas normas y cómo llegan a ser aceptadas por los participantes es una parte importante del enfoque inferencialista que estamos siguiendo, pero que hemos elegido no desarrollar en este trabajo, sino que dejaremos 
para investigaciones posteriores, aunque sí hemos destacado la existencia de distintos tipos de normas, su carácter contingente y la falta de arbitrajes claros. La revisión entre colegas y las evaluaciones externas son posibles porque existen normas. La revisión entre colegas es la clave sobre la que pivota el desarrollo de una teoría científica y por ello es importante ser consciente de las dificultades con las que se encuentra.

El lado más irracional de la ciencia está precisamente en el exceso de tenacidad que muestran los científicos y el resto de agentes involucrados en ella frente a las evidencias empíricas y analíticas que se les presentan. Pero al mismo tiempo, no todos los participantes son tenaces en las mismas creencias a la vez, ni con la misma intensidad. Las inferencias más convincentes pueden ir ganando adeptos en aquellos que están dispuestos a cambiar parte de sus creencias afectadas. Las situaciones de equilibrio, aquellas en las que cada uno cree estar en su mejor opción, van cambiando, y en definitiva, la ciencia en su conjunto puede ir progresando racionalmente, superando las ineficiencias. La ciencia no es el fundamento inapelable para tomar las decisiones, aunque es quizás la mejor guía de la que disponemos.

\section{REFERENCLAS}

Aldred, Jonathan. 2009. Ethics and climate change cost-benefit analysis. Stern and After. Environmental Economy and Policy Research Working Papers 44. University of Cambridge.

Betz, Gregor. 2009. Underdetermination, model ensembles and surprises on the epistemology of scenario-analysis in climatology. Journal for General Philosophy of Science 40 (1): 3 - 21

Brandom, Robert. 1994. Making it explicit. Reasoning representing and discursive commitment. Cambridge MA: Harward University Press.

-. 2000. Articulating Reasons. An introduction to inferentialism. Cambridge MA: Harward University Press.

Koktvedgaard, Hanne. "Cases considered in 2002" in Annual report 2002 of The Danish Comittee on Scientific Dishonesty (Danish Agency for Science, Technology and Innovation, 2003)

Esper, J. et al., 2002. Low-frequency signals in long-tree chronologies for reconstructing past frequency variability. Science 295: 2250 - 2253.

Franz Wendy. 2007. The developing of an agenda for climate change: Connecting science to policy. Discussion paper E-97. Harvard University Press.

Gavin, Neil et al., 2009. Climate Change and the media in Britain: SAGE publications. http.//www.sagepublications.com

Gilland, Tony. Digging the roots of the IPCC. http://www.spiked-online.com (Acceso en Junio 2007)

Feyerabend, Paul. 1975. Against method. London: NLB

Authority of House of Commons, eds. 2010. The disclosure of climate data from the Climatic Research Unit at the University of East Anglia. London: The Stationery Office Limited.

Jansen, E. et al., 2007. "Paleoclimate. The Physical science basis" in Contribution of Working group I to the Forth Assessment report of the IPCC. Cambridge: Cambridge University Press.

Kuhn, Thomas S., 1962. The Structure of scientific revolutions. Chicago: The University of Chicago Press.

-. 1977. Objetivity, Value Judgments and Theory Choice. The Essential Tension. Chicago: The University of Chicago Press.

Lakatos, Imre. 1978. The Methodology of Scientific Research Programmes: Philosophical Papers Volume 1: Cambridge University Press.

Lomborg, B., 2001. The skeptical ecologist. Cambridge: Cambridge University Press.

Monbiot, George. 2009. Global Warming rigged? Here is the mail Id need to see. Guardian.co.uk $23 / 11 / 2009$

—. 2008. Un intercambio de almas. Una crítica al informe Stern. The Guardian 4/2008.

Nature, eds. 2009. A crisis of confidence. Nature 457 
Nordhaus, William, 2007. The Stern Review of the Economic of Climate Change. Journal of Economic Literature 45 (3): 686-702.

Popper, Karl. 1934 (ed. inglesa 1959). The Logic of Scientific Discovery. Routledge, London.

-. 1963. Conjectures and refutations. The Growth of scientific Knowledge. Routledge. London.

Rörsch, A. 2008. Good scientific practice and the Lomborg case affair in Denmark: Leyden University Press.

Russell, Muir et al. 2010. Report of the independent climate change e-mail review. East Anglia University. http:/ / www. cce-review.org (Acceso en Julio 2010)

Schneider, Stephen. 1976. The Genesis strategy. Climate and global Survival. New York. Plenum Press.

Stern, Nicholas. 2006. Stern review of the economics of climate change. London. HM Treasury.

Weart, Spencer, 2004. The discovery of global warming: Harvard University Press.

Weitzman, Martin. 2007. The Stern Review of the economics of Climate Change. Journal of Economic Literature 45 (3): 686-702.

Zamora Bonilla, Jesús P. 2006 a. Science as a persuasion game. An Inferentialist Approach. Episteme 191.

—. 2006b. Science studies and the theory of games Perspectives on Science, Episteme 14.4:525-557.

- 2009. What Games Do Scientists Play? Rationality and Objectivity in a Game-Theoretic Approach to the Social Construction of Scientific Knowledge. EPS: Launch of the European Philosophy of Science Association, Springer, Amsterdam, 323-332.

Zia, Asim, and Todd, Anne. 2010. Evaluating the effects of ideology on public understanding of climate change science: How to improve communication across ideological divides? Public Understanding of Science 19(6): 743-761

Leonardo Monzonís Forner es Ingeniero de Caminos, Canales y Puertos, y desde 1990 es ingeniero del estado. A partir de 1994 ejerce su actividad en el campo de la ingeniería marítima en la Dirección General de Sostenibilidad de la Costa y del Mar. Licenciado en Filosofía desde el año 2004, obtuvo la suficiencia investigadora en el año 2006, y redacta actualmente su tesis doctoral en el Departamento de Historia, Lógica y Filosofía de la Ciencia, de la UNED.

Dirección: Facultad de Filosofía, UNED. 28040 Madrid, España. E-mail: LMonzonis@magrama.es

Jesús Zamora Bonilla es catedrático de la UNED, y autor de numerosos libros y artículos de filosofía. Algunas obras recientes son La caverna de Platón y los cuarenta ladrones (Le Pourquois-pas?, 2011), The SAGE Handbook of Philosophy of Social Science (coeditor, con Ian Jarvie; SAGE, 2011), "The economics of scientific knowledge" (en Handbook of Philosophy of Economics, Elsevier, 2012), o "Why are good theories good?" (Synthese, aceptado).

DiRección: Facultad de Filosofía, UNED. 28040 Madrid, España. E-mail: jpzb@fsof.uned.es 